
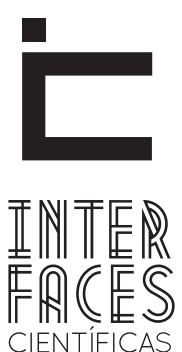

SAÚDE E AMBIENTE

ISSN IMPRESSO 2316-3313

E-ISSN 2316-3798

DOI - 10.17564/2316-3798.2017v6n1p9-18

\title{
EFEITO DO PILATES SOLO NA FORÇA ABDOMINAL E NA POSTURA DE MULHERES IDOSAS COM LOMBALGIA ${ }^{1}$ \\ ABDOMINAL STRENGTH OF ELDERLY PRACTITIONERS OF PILATES \\ EFECTO DEL PILATES EN El SUELO EN LA FUERZA ABDOMINAL Y EN LA POSTURA DE MUJERES EN TERCERA EDAD CON LUMBAGO
}

Leliz Cristina Sampaio Queiroz ${ }^{1}$
Sonia Maria Marques Gomes Bertolini ${ }^{3}$
Eraldo Schunk Silva ${ }^{2}$

Rose Mari Bennemann ${ }^{4}$

\section{RESUMO}

As práticas corporais e as atividades físicas têm sido reconhecidas como grandes aliadas do envelhecimento saudável, além de serem temas prioritários da atual Política Nacional de Promoção da Saúde. 0 método Pilates foi proposto como uma alternativa de atividade física para a população idosa, uma vez que visa o alongamento e o fortalecimento muscular simultaneamente, podendo melhorar parâmetros motores e consequentemente a qualidade de vida na terceira idade. Assim, o objetivo do estudo foi analisar a influência dos exercícios de Pilates no solo sobre a força da musculatura abdominal, a postura e a dor lombar de mulheres idosas. 0 estudo foi do tipo quantitativo

1. Artigo baseado na Dissertação de Mestrado de Leliz Cristina Sampaio Queiroz intitulada "Força abdominal, dor lombar e massa muscular em ido- analítico, com coleta de dados primários e amostra por conveniência. Participaram do estudo 43 idosas que foram submetidas a 39 sessões de exercícios de Pilates no solo. 0 fortalecimento abdominal das idosas foi avaliado pelas repetições no teste de força abdominal com banco, durante dois minutos. As voluntárias do estudo obtiveram aumento significativo da força da musculatura abdominal $(p<0,003)$, diminuição das assimetrias corporais $(p<0,001)$ e redução da dor $(p<0,001)$. Conclui-se que os exercícios de Pilates no solo são eficazes para melhorar o trabalho da musculatura abdominal, diminuir os desvios posturais e a dor lombar de mulheres idosas.

sos praticantes de Pilates solo", defendida no Programa de Mestrado Promoção da Saúde do Centro Universitário de Maringá. 


\section{PALAVRAS-CHAVE}

Exercício Físico. Idoso. Músculos Abdominais.

\section{ABSTRACT}

Bodily practices and physical activities has been recognized as great allies of healthy aging, as well as being priority issues of the current National Health Promotion Policy. The Pilates method was proposed as an alternative to physical activity for older adults, since intended stretching and strengthening muscle simultaneously, thus improving engine parameters and consequently the quality of life in aging. In this sense, the objective of the study was to analyze the influence of Pilates exercises on the ground on the strength of the abdominal muscles, posture and back pain elderly. The study was analytical quantitative, with primary data collection and sample convenience. The study included 43 elderly women who underwent 39 sessions of Pilates exercises on the ground. Abdominal

\section{RESUMEN}

Las prácticas corporales y las actividades físicas han sido reconocidas como grandes aliadas del envejecimiento saludable, además de ser temas prioritarios de la actual política brasileña de promoción a la Salud. El método Pilates fue propuesto como una alternativa de actividad física para la población de adultos mayores, una vez que esta actividad permite el alargamiento y el fortalecimiento muscular simultáneamente, lo que puede mejorar parámetros motores y consecuentemente la calidad de vida en esta población específica. Así, el objetivo del estudio fue analizar la influencia de los ejercicios de Pilates en el suelo sobre la fuerza de la musculatura abdominal, la postura y el dolor lumbar de mujeres adultas mayores. El estudio fue de tipo cuantitativo analítico, con recolección de datos
Strengthening older was estimated by repetitions of the abdominal strength test bench for two minutes. The subjects in the study had a significant increase in the strength of the abdominal muscles $(p<0.003)$ decrease in body asymmetries $(p<0.001)$ and reduced pain $(p<0.001)$. It follows that Pilates exercises on the ground are effective to improve the working of the abdominal muscles, postural deviations and reduce lower back pain elderly.

\section{KEYWORDS}

Physical exercise. Old man. Abdominal muscles. primarios a partir de una muestra por conveniencia. Participaron del estudio 43 mujeres adultas mayores que fueron sometidas a 39 sesiones de ejercicios de Pilates en el suelo. El fortalecimiento abdominal de las mujeres fue evaluado por las repeticiones de la prueba de fuerza abdominal con banco, durante dos minutos. Las voluntarias del estudio obtuvieron un aumento significativo de la fuerza de la musculatura abdominal $(p<0,003)$, disminución de las asimetrías corporales $(p<0,001)$ y reducción del dolor $(p<0,001)$. Se concluye que los ejercicios de Pilates en el suelo son eficaces para mejorar el trabajo de la musculatura abdominal, disminuir las desviaciones posturales y el dolor lumbar de mujeres adultas mayores. 


\section{PALABRAS CLAVE}

Ejercicio físico, Adulto mayor, Músculos abdominales.

\section{INTRODUÇ̃̃O}

Com o envelhecimento, a degeneração muscular e osteoarticular é natural e inevitável, o que pode ocasionar em alguns indivíduos a dor lombar que é responsável por significativa demanda de incapacidade funcional e serviços em saúde coletiva. Esta sintomatologia é considerada uma das dores mais relatadas por idosos, perdendo apenas para a dor de cabeça (KLEINPAUL et al., 2008).

Práticas corporais e exercícios físicos são reconhecidos como grandes aliados do envelhecimento mais saudável. Lee e outros autores (2014) relataram que os exercícios de fortalecimento dos músculos abdominais e pélvicos são amplamente utilizados como um meio para estabilizar o tronco, proporcionando a melhoria da dor lombar crônica e da postura.

De acordo com Araújo e outros autores (2010), os exercícios de Pilates são capazes de aumentar a flexibilidade dos segmentos corporais, o alinhamento postural e a coordenação motora, além de intensificar a força muscular. 0 método caracteriza-se por exercícios que envolvem contrações concêntricas, excêntricas e principalmente isométricas, com ênfase nos músculos abdominais, multífidos e do assoalho pélvico, os quais são responsáveis pela estabilização estática e dinâmica do corpo (MARES et al., 2012).

0 referido método consiste ainda de movimentos corporais que envolvem condicionamento físico e mental, com vasta variação de exercícios de baixo impacto articular, concentração, equilíbrio, relaxamento, controle corporal e respiração (MIRANDA; MORAIS, 2009). Estes movimentos corporais podem ser realizados em aulas com aparelhos e aulas no solo, estas últimas também denominadas The Mat (MARÉS et al., 2012).

Os exercícios realizados no solo têm apresentado benefícios semelhantes aos praticados com ajuda de aparelhos criados por Josephe Pilates no início dos anos 1920 do século passado. Nestes exercícios são utilizadas estruturas de madeira e metais com molas e tiras de couro, cama e empunhaduras. No solo, por sua vez, o praticante precisa usar a força do próprio corpo para realizar os exercícios, o que faz com alguns exercícios se tornem mais pesados (FORCECIA, 2012).

Com vistas à importância de se manter uma vida saudável durante o processo de envelhecimento e a grande procura por essa modalidade de atividade física em todas as faixas etárias, o objetivo destee estudo foi analisar a influência dos exercícios de Pilates no solo sobre a força da musculatura abdominal, postura e dor lombar de mulheres idosas.

\section{MÉTODOS}

Trata-se de um estudo do tipo ensaio aleatório, realizado por meio de um programa de intervenção com exercícios do Método Pilates no solo, com avaliações em momentos pré e pós-intervenção, submetido e aprovado pelo Comitê de Ética em Pesquisas com seres Humanos do Centro Universitário de Maringá (parecer no 959.474).

Participaram do estudo mulheres com idade entre 60 a 88 anos, frequentadoras do Centro Cultural e Social São Francisco Xavier e Serviço de Convivência para Idosos “Irmã Clara Kô", localizados no município de Maringá, Estado do Paraná.

Foram coletados dados demográficos, por meio de questionário, constando informações referentes a identificação pessoal, profissão e data de nascimento. Todas as idosas que aceitaram participar da 
pesquisa assinaram o termo de consentimento livre e esclarecido e foram informadas detalhadamente sobre os procedimentos utilizados, seguindo a resolução específica do Conselho Nacional de Saúde ( $n^{\circ}$ 466/2012).

Foram incluídas, partindo-se do universo apresentado, todas as idosas que aceitaram participar do estudo, as quais apresentaram atestado médico para a prática de exercícios físicos e disponibilidade de tempo para participarem da pesquisa. Foram excluídos idosos do sexo masculino e idosas com problemas motores/físicos que impedissem a prática de exercícios físicos.

A avaliação da força muscular abdominal foi realizada por meio do teste abdominal de dois minutos, conforme o protocolo de Knudson e Johnston (1995). Neste teste, o indivíduo deve ficar posicionado em decúbito dorsal sobre um colchonete, com flexão de joelhos e quadris, estando as pernas (gastrocnêmios) apoiadas sobre um banco com altura de $46 \mathrm{~cm}$, os braços cruzados à frente do corpo e as mãos fixadas nos ombros do lado contrário.

O movimento de flexão anterior da coluna foi realizado até os cotovelos encontrarem a porção anterior da coxa (quadríceps), sendo que uma repetição foi contabilizada quando a idosa retornava à posição inicial. Para avaliar o número de repetições de flexões abdominais somaram-se o número de repetições do tronco. Entende-se que quanto maior o número de repetições alcançadas no período de dois minutos, melhor a força abdominal da idosa.

A avaliação da dor foi mensurada pela escala visual analógica (EVA) para dor (Visual Analogue Scale - VAS), um instrumento unidimensional para a avaliação da intensidade da dor. A EVA é a imagem de uma linha com as extremidades numeradas de 0 a 10, onde em uma extremidade da linha é marcada "nenhuma dor" e na outra "pior dor imaginável" (CIENA et al., 2008). Assim cada participante que marcou na linha a dor presente naquele momento.

0 procedimento de intervenção foi feito em grupos, onde uma profissional devidamente treinada fi- cou responsável por aproximadamente quinze alunas. As idosas foram submetidas a aulas práticas de Pilate Solo, nível iniciante, onde executaram exercícios sobre solo protegido, forrado com material apropriado para melhor conforto aos praticantes.

A avaliação postural foi mensurada com auxílio de um Simetrógrafo portátil de parede junto a idosa avaliada. A comparação foi por meio do "Teste de New Tork” (ADAMS et al., 1985). Este teste objetiva identificar a instalação de desalinhamento nas estruturas corporais por meio de parâmetros já estabelecidos, baseando-se na comparação da postura estática em um gráfico conhecido como New York Posture Rating.

0 referido teste classifica a postura estática em três níveis: normal, moderado e acentuado. A idosa foi colocada em posição ortostática, vestindo roupas leves e confortáveis. Existem 13 itens a analisar, sendo seis na posição de costas e sete de perfil. Cada item foi atribuído a uma nota (1, 3 e 5). A nota global menor ou igual a 39 corresponde a desnivel posturais (LONGO, 2008).

As práticas foram realizadas no período da manhã, compreendendo um período de 12 semanas, perfazendo um total de 39 sessões. Ainda sendo a duração de cada sessão (aula) 40 minutos/dia, três vezes por semana, conforme preconizado por Williams e outros autores (2005) e Kuhnert (2002).

Foram utilizados materiais auxiliares como: bolas de Pilates e elásticos. Cada sessão foi executada com exercícios de alongamentos, de estabilização da pelve, fortalecimento do abdômen e dos membros superiores e inferiores, exercícios de respiração, além de noções de concentração, equilíbrio, controle corporal e relaxamento. A progressão do protocolo foi baseada no aumento da dificuldade por meio de variações de posturas intermediárias, para cada exercício.

No primeiro momento foram abordados os princípios do método como: concentração, respiração, centralização, fluidez, precisão e controle. Nas 10 primeiras aulas, foram realizadas 05 repetições de cada exercício; na $11^{\mathrm{a}}$ à $39^{\mathrm{a}}$ aula, foram realizadas 10 repetições de cada exercício (QUADRO 1). 
Quadro 1 - Protocolo de exercícios de Pilates Solo

\begin{tabular}{|c|c|}
\hline Exercícios & Objetivos \\
\hline $\begin{array}{l}\text { Spine Stretch } \\
\text { Forward }\end{array}$ & $\begin{array}{l}\text { Alongar a cadeia posterior e mobili- } \\
\text { zação da coluna. }\end{array}$ \\
\hline $\begin{array}{l}\text { Rolling like a } \\
\text { Ball }\end{array}$ & $\begin{array}{l}\text { Fortalecer os músculos reto abdo- } \\
\text { minal, oblíquo externo e mobilizar } \\
\text { a coluna. }\end{array}$ \\
\hline Swan & $\begin{array}{l}\text { Alongar a cadeia anterior do tronco } \\
\text { e mobilizar a coluna; fortalecimen- } \\
\text { to do peitoral maior, tríceps bra- } \\
\text { quial, ancôneo e deltóide anterior. }\end{array}$ \\
\hline Teaser & $\begin{array}{l}\text { Fortalecer os músculos reto abdo- } \\
\text { minal e obliquo externo. }\end{array}$ \\
\hline Saw & $\begin{array}{l}\text { Alongar os músculos rotadores } \\
\text { do tronco, isquiotibiais, quadrado } \\
\text { lombar, fortalecimento dos múscu- } \\
\text { los reto abdominal, obliquo externo } \\
\text { e interno. }\end{array}$ \\
\hline $\begin{array}{l}\text { Shoulder } \\
\text { Bridge }\end{array}$ & $\begin{array}{l}\text { Fortalecer os músculos quadríceps, } \\
\text { glúteos e musculatura posterior da } \\
\text { perna. }\end{array}$ \\
\hline $\begin{array}{l}\text { Single Leg } \\
\text { Stretch }\end{array}$ & $\begin{array}{l}\text { Fortalecer a musculatura abdomi- } \\
\text { nal; alongar glúteos e musculatura } \\
\text { da coluna lombar. }\end{array}$ \\
\hline Criss Cross & $\begin{array}{l}\text { Fortalecer os músculos reto abdo- } \\
\text { minal, obliquo externo e interno. }\end{array}$ \\
\hline Leg Pull Front & $\begin{array}{l}\text { Fortalecer os músculos peitoral } \\
\text { maior, tríceps braquial, ancôneo, } \\
\text { deltoide anterior, glúteo máximo, } \\
\text { bíceps femoral, semimembranáceo } \\
\text { e paravertebrais. }\end{array}$ \\
\hline Roll-over & $\begin{array}{l}\text { Fortalecer os músculos obliquo } \\
\text { externo e reto femoral }\end{array}$ \\
\hline Swimming & $\begin{array}{l}\text { Fortalecer os músculos paraverte- } \\
\text { brais, deltóide posterior e médio, } \\
\text { glúteo máximo, bíceps femoral, } \\
\text { semitendíneo e semimembranáceo. }\end{array}$ \\
\hline
\end{tabular}

\begin{tabular}{|l|l|}
\hline \multicolumn{1}{|c|}{ Exercícios } & \multicolumn{1}{c|}{ Objetivos } \\
\hline Side Plank & $\begin{array}{l}\text { Fortalecer a musculatura estabili- } \\
\text { zadora da coluna. }\end{array}$ \\
\hline $\begin{array}{l}\text { One Leg } \\
\text { Circle }\end{array}$ & $\begin{array}{l}\text { Trabalhar o alinhamento e o contro- } \\
\text { le do tronco; alongar a musculatura } \\
\text { posterior; estabilizar a coluna. }\end{array}$ \\
\hline $\begin{array}{l}\text { Hamstring } \\
\text { Series (deita- }\end{array}$ & $\begin{array}{l}\text { Fortalecer e alongar isquiotibiais; } \\
\text { fortalecer adutores; estimular a } \\
\text { espuma) }\end{array}$ \\
$\begin{array}{l}\text { propriocepção para o controle do } \\
\text { tronco e alongamento axial; alon- } \\
\text { gar o eixo longitudinal associado à } \\
\text { estabilização; estimular o controle } \\
\text { da pelve e dissociação de quadril. }\end{array}$ \\
\hline
\end{tabular}

Fonte: Forcecia (2012).

Os dados foram descritos por meio de tabelas de frequências simples e cruzadas, média, desvio-padrão (DP) e coeficiente de variação (CV). A diferença entre as medidas pré e pós-programa de intervenção, para as variáveis Força abdominal, Dor e postura foi testada por meio do teste para amostras pareadas de Wilcoxon (Wilcoxon Signed Rank Test).

A decisão de se rejeitar $\mathrm{H}_{0}$ ou não foi tomada, considerando-se um nível de confiança de $95 \%(\alpha=0,05)$, ou seja, p-valor menor que 0,05 . Os dados foram analisados no Programa Statistical Analysis Software (SAS, version 9.0), a partir de uma base de dados construída por meio de uma planilha eletrônica do programa Microsoft Office Excel, versão 2007.

\section{RESULTADOS}

Do total de 65 voluntárias que iniciaram o protocolo de intervenção, apenas 43 idosas $(66,2 \%)$ completaram efetivamente as 39 sessões. A média, o desvio padrão e coeficiente de variação da idade, estatura, peso das participantes do estudo encontram-se na Tabela 1. 
Tabela 1 - Média, Desvio padrão e Coeficiente de variação da idade, estatura, peso de idosas praticantes de exercícios de Pilates Solo. Maringá, Paraná, 2016.

\begin{tabular}{cccc}
\hline Variáveis & Média & DP & CV \\
\hline Idade $($ anos $)$ & 71,23 & 6,22 & 8,73 \\
\hline Estatura $(\mathrm{m})$ & 1,55 & 0,07 & 4,51 \\
\hline Peso $(\mathrm{kg})$ & 64,50 & 12,6 & 19,58 \\
\hline
\end{tabular}

$\mathrm{DP}=$ desvio padrão; $\mathrm{CV}=$ coeficiente de variação.

Fonte: Dados da pesquisa.

A média de repetições abdominais pré-intervenção, segundo o teste de força abdominal, foi de 31,51 $\pm 15,12$ com mínimo de zero e máximo de 59 movimentos. Após a intervenção, o número de repetições variou de zero a 73, com média de 35,58 $\pm 17,21$. Conforme mostrado na Tabela 2, os valores obtidos pelas participantes no teste de força abdominal, antes e após o protocolo de intervenção foram diferentes, apresentaram diferenças estatisticamente significantes $(p=0,003)$.

A média do nível de dor lombar pré e pós-intervenção foi de $3,81 \pm 2,91$ e de $1,56 \pm 2,00$, respectivamente, indicando redução significativa desse sintoma (TABELA 2). Quanto à postura, a média antes e após a intervenção foi de $38,77 \pm 7,85$ e $44,07 \pm 8,07$, respectivamente, o que também mostrou uma significativa melhora nos desníveis posturais (TABELA 2).

Tabela 2 - Média, Desvio-padrão (DP) e Coeficiente de variação (CV), nos momentos pré e pós protocolo de intervenção, para as variáveis peso, força abdominal, dor lombar e postura em idosas. Maringá, Paraná, 2016

\begin{tabular}{|c|c|c|c|c|c|c|c|}
\hline \multirow[b]{2}{*}{ Variáveis } & \multicolumn{3}{|c|}{ Pré intervenção } & \multicolumn{3}{|c|}{ Pós intervenção } & \multirow[b]{2}{*}{ Valor de $p$} \\
\hline & Média & DP & CV & Média & DP & $\mathrm{CV}$ & \\
\hline Peso $(\mathrm{kg})$ & 64,2 & 12,53 & 19,52 & 64,50 & 12,67 & 19,64 & 0,807 \\
\hline Força abdominal & 31,51 & 15,12 & 47,98 & 35,58 & 17,21 & 48,36 & $0,003 *$ \\
\hline Dor lombar & 3,81 & 2,91 & 76,39 & 1,56 & 2,00 & 128,59 & $<0,001^{*}$ \\
\hline Postura & 38,77 & 7,85 & 20,25 & 44,07 & 8,07 & 18,30 & $<0,001^{*}$ \\
\hline
\end{tabular}

* Significativo ao nível de confiança $95 \%$.

Fonte: Dados da pesquisa.

\section{DISCUSSÃO}

O aumento da capacidade de contração da musculatura abdominal observada neste estudo demonstra o efeito positivo do conjunto de 39 sessões de exercícios de Pilates. Este fato é de grande relevância devido o papel desempenhado por estes músculos na manutenção da integridade da região lombar da coluna vertebral, principalmente em se tratando de população idosa e feminina.

Estudo realizado por Pinheiro e outros autores (2014) evidenciou já na $12^{a}$ sessão de exercícios de Pilates no solo, melhora na eficácia da contração do músculo transverso do abdome, bem como na musculatura paravertebral lombar em idosas. Vale destacar que a média de idade das idosas do presente estudo era maior ( $71,23 \pm 6,22$ anos) e para aplicação do teste considerou-se o grupo de músculos abdominais e não um músculo específico, como o transverso do abdome. A escolha pelo grupo muscular justifica-se pelo fato do Método Pilates ser caracterizado por movimentos eficientes da musculatura abdominal, paravertebral e da região glútea (MARÉS et al., 2012).

A flexibilidade não se configurou em objeto de investigação da presente pesquisa, mas ganhos de flexibilidade de mulheres sedentárias acompanhados por aumento de força abdominal foram observados por Sekendizet e outros autores (2007) em estudo anterior.

A dor lombar em muitos casos está associada a fraqueza muscular e a má postura. Nesse sentido, exercícios que desenvolvam força muscular favorecem a proteção das articulações, podendo prevenir lesões li- 
gamentares, dores nas costas, além de ajudar a manter uma boa postura (NAHAS, 2013). A variável dor também apresentou melhora significativa nesta pesquisa realizada no Estado do Paraná, Brasil. Este fato mostra mais uma vez que mesmo em indivíduos com idade mais avançada, em que muitos problemas da coluna vertebral já são estruturados, o fortalecimento da musculatura pode promover um melhor alinhamento corporal.

Pesquisa realizada com mulheres empresárias que sentiam dores crônicas na região lombar da coluna vertebral também mostrou resultados positivos com o Método Pilates (LEE et al., 2014). Os autores ainda compararam os resultados dos exercício em aparelhos e no solo, sendo estes últimos mais eficazes.

No estudo de caso com um paciente portador de espondilolistese traumática de L4-L5 e que apresentava quadro estável; Oliveira e outros autores (2013) verificaram que o método Pilates foi eficiente no aumento da força da musculatura abdominal e paravertebral, bem como melhorou a flexibilidade e diminuiu a dor.

0 presente estudo também demonstrou melhora significativa na postura corporal $(p<0,001)$. Estes achados corroboram o estudo de Nunes Junior e colaboradores (2008) os quais encontraram melhora significante no alinhamento postural de voluntários de meia idade e idosas saudáveis, após 36 sessões de Pilates, 3 vezes por semana, com duração de uma hora. Os efeitos posturais do Pilates poderiam ser atribuídos aos aumentos da resistência e força muscular, como verificado por Ferreira e outros autores (2007).

Os resultados da pesquisa realizada por Carvalho (2009), com objetivo de investigar a eficácia do método Pilates sobre a força abdominal, flexibilidade e postura de mulheres de diferentes faixas etárias, também indicou resultados positivos, principalmente, para mulheres da terceira idade.

Ainda, Sinzato e outros autores (2013), com uma amostra de mulheres mais jovens e com 20 sessões de Pilates concluíram que o referido método pode ser capaz de gerar ganhos significantes na flexibilidade articular, entretanto os autores declaram que a quantidade de sessões não foi suficiente para provocar adaptações posturais.
Estudo realizado por Rodrigues e colaboradores (2010) com objetivo de avaliar o efeito do Método Pilates na autonomia funcional de idosas, apontou após 12 semanas de exercícios melhora expressiva no desempenho funcional das 52 voluntárias. Considerando que um dos testes utilizados refere-se a levantar-se da posição de decúbito dorsal, pode-se encontrar similaridades com a presente pesquisa. Ainda quanto a variável peso corporal os resultados não mostraram indícios de que o Pilates favoreça a sua redução.

\section{CONCLUSÃO}

A partir da presente pesquisa pode-se concluir que a prática do Pilates Solo é eficaz para o fortalecimento da musculatura abdominal, diminuição da dor lombar e melhora da postura de idosas. Tais fatos nos atentam para a importância da prática dessa atividade uma vez que o fortalecimento da musculatura abdominal em idosas está intimamente ligado ao equilíbrio e distribuição dos esforços na execução de atividades diárias, não sobrecarregando outras musculaturas do corpo.

Outra questão importante é ao fato da dor ser a principal queixa e causa de limitações funcionais entre os idosos, afetar um número grande de pessoas que sofrem desse mal e ser uma das principais características do processo de envelhecimento. Sendo assim, o resultado significativo na diminuição da dor lombar após realização de exercícios do método Pilates solo, mostrou que a prática dessa atividade contribui para a qualidade de vida dessas pessoas.

Além disso, os resultados positivos quanto à postura corporal das idosas participantes da pesquisa, nos mostra a importância de se manter ativo e assim melhorar os desvios posturais, fato muito observado nas pessoas com idade avançada.

Sem dúvida, os resultados significativos da pesquisa realizada com idosas paranaenses nos fazem refletir e concordar que manter uma vida ativa durante todas as fases etárias é fundamental para a qualidade de vida 
das pessoas com mais de 60 anos. No entanto, sugerem-se pesquisas futuras com amostras populacionais maiores, bem como com idosos de ambos os sexos.

\section{REFERÊNCIAS}

\section{ADAMS, R.C. et al. Esportes e exercícios para}

deficiente físico. 3.ed. São Paulo: Manole Ltda.,1985.

ARAÚJO, M.E.A. et al. Redução da dor crônica associada à escoliose não estrutural, em universitárias submetidas ao método Pilates. Motriz Rev. Ed. Fís., v.16 n.4, p.958-966, 2010.

CARVALHO, C.M. Eficácia dos exercícios do método pilates na flexibilidade, força muscular e postura de mulheres em idades diferenciadas. 2009. $94 \mathrm{f}$. Dissertação (Mestrado) - Universidade Castelo Branco, Rio de Janeiro, 2009.

FERREIRA, C.B. et al. 0 método Pilates ${ }^{\oplus}$ sobre a resistência muscular localizada em mulheres adultas. Motricidade, v.3, n.4, p.76-81, 2007.

FORCECIA. Diferença do Pilates no Solo e Pilates nos Aparelhos. Rev. Pilates. [On-line]. Disponível em: <http://revistapilates.com.br/2012/03/13/diferencade-pilates-no-solo-e-pilates-nos-aparelhos>. Acesso em: 27 out. 2014.

KLEINPAUL, J.F. et al. Dor lombar e exercício físico: Uma revisão. Rev. Dig. Buenos Aires. 13.ed. Ano 13, dez. 2008. Disponível em: <http://www.efdeportes. com/efd127/dor-lombar-e-exercicio-fisico.htm> Acesso em: 27 out. 2014.

KUHNERT, C. Um corpo perfeito com pilates. Vitória Régia: São Paulo, 2002.

LEE, C.; HYUN, J.; KIM, S. G. Influence of Pilates Mat and Apparatus Exercises on Pain and Balance of
Businesswomen with Chronic Low Back Pain. J Phys Ther Sci. v.26, n.4, p.475-477, 2014.

MARÉS, G. et al. A Importância da Estabilização Central no Método Pilates: Uma Revisão

Sistemática. Revista Fisioter. Mov., v.25, n.2, p.445-451, 2012.

MIRANDA, L.B.; MORAIS, P.D.C. Efeito do Método Pilates sobre a composição corporal e flexibilidade. Rev. Bras.

Prescr. Fisiol. Exerc., v.3, n.13, p.16-21, 2009.

NAHAS, M.V. Atividade física, saúde e qualidade de vida. Conceitos e sugestões para um estilo de vida ativo. 6.ed. Londrina: Midiograf, 2013.

NUNES Jr., P.C. et al Os efeitos do Método Pilates no alinhamento postural: estudo piloto. Fisioterapia Ser, v.3, n.4, p.210- 215, 2008.

OLIVEIRA, L.C. et al. O método Pilates no tratamento de espondilolistese traumática em L4-L5: estudo de caso. Fisioter. mov. v.26, n.3, p.623-629, 2013.

PINHEIRO, K.R.G. et al Influence of pilates exercises on soil stabilization in lumbar muscles in older adults. Rev. bras. cineantropom. desempenho hum. v.16, n.6, p.648-657, 2014.

RODRIGUES, B.G.S. et al Autonomia funcional de idosas praticantes de Pilates. Rev. Fisioterap. Pesq., v.17, n.4, p.300-305, 2010.

SEKENDIZ, B. et al Effects of Pilates exercise on trunk strength, endurance and flexibility in sedentary adult females. Jornal Bodyw Mov Ther. v.11, n.4, p.318-326, 2007.

SINZATO, C.R. et al Efeitos de 20 sessões do método Pilates no alinhamento postural e flexibilidade de mulheres jovens: estudo piloto. Fisioter Pesq. v.20, n.2, p 143-150, 2013. 
WILLIAMS, S.; JASEN, D. Para começar a praticar

pilates. São Paulo: Publifolha, 2005.

Recebido em: 10 de Maio de 2017

Avaliado em: 11 de Maio de 2017

Aceito em : 12 de Maio de 2017
1. Mestre em Promoção da Saúde Pelo Centro Universitário de Maringá UniCesumar. Email: cristinaleliz@yahoo.com.br

2. Professor do Programa de Mestrado em Bioestatística da Universidade Estadual de Maringá. Email: eraldoschunk@gmail.com

3. Professora do Programa de Mestrado em Promoção da Saúde do Centro Universitário de Maringá - UniCesumar; Professora Associada do Centro de Ciências Morfológicas - Universidade Estadual de Maringá. Email: sonia.bertolini@unicesumar.edu.br

4. Professora do Programa de Mestrado em Promoção da Saúde do Centro Universitário de Maringá - UniCesumar. Email: rose.bennemann@gmail.com 